\title{
Many-body effects on bandgap shrinkage, effective masses and alpha factor
}

\author{
Jian-Zhong $\mathrm{Li}^{\dagger}$ and C. Z. Ning \\ NASA Ames Research Center, M/S N229-1, Moffett Field, CA94035. L.S.A.
}

\begin{abstract}
Many-body Coulomb effects influence the operation of quantum-well (QW) laser diode (LD) strongly. In the present work, we study a two-band electron-hole plasma (EHP) within the Hatree-Fock approximation and the single plasmon pole approximation for static screening. Full inclusion of momentum dependence in the many-body effects is considered. An empirical expression for carrier density dependence of the bandgap renormalization (BGR) in an $8 \mathrm{~nm} \mathrm{GaAs} / \mathrm{Al}_{0.3} \mathrm{Ga}_{0.7} \mathrm{As}$ single $\mathrm{QW}$ will be given, which demonstrates a non-universal scaling behavior for quasitwo-dimension structures, due to size-dependent efficiency of screening. In addition. effective mass renormalization (EMR) due to momentum-dependent self-energy many-body correction, for both electrons and holes, is studied and serves as another manifestation of the many-body effects. Finally, the effects on carrier density dependence of the alpha factor is evaluated to assess the sensitivity of the full inclusion of momentum dependence.
\end{abstract}

Keywords: quantum well, laser diode, many-body effect, bandgap renormalization. effective mass, alpha factor

\section{INTRODUCTION}

Performance of semiconductor quantum-well laser diode is influenced by many-body effects in an important way. ${ }^{1.2}$ At high excitation level semiconductors are described as an electron-hoel plasma. Coulomb interaction among carriers results in several overall modifications in the semiconductor LD. First of all. energr dispersion of carriers is changed due to exchange and correlation effect. A well-known example is BGR. Also, the interaction leads to strong screening and scatterings at lasing carrier density. It is the ultrafast scattering timescale that allows the assumption of quasiequilibrium for the carrier distributions. In addition, light-matter interaction is renormalized by carrier-carrier interaction, which is exemplified by the Coulomb enhancement effect in the absorption spectrum of a semiconductor.

Bandgap renormalization in semiconductor QW' structrues has been extensively investigated, both theoretically and experimentally. ${ }^{13-15}$ After summarizing photoluminescence (PL) data for several semiconductor QW systerns. Tränkle et. al. ${ }^{7,8}$ seemingly revealed a universal scaling law for BGR in quasi-2D semiconductor QW structures, by scaling with 2D excitonic Rydberg and Bohr radius. Jones et. al. ${ }^{15}$ used the quasi-2D excitonic Rydberg and Bohr radius to scale their magnetoluminescence data for a series of $8 \mathrm{~nm} n$-type GaAs/AlGaAs single QWs, and found that their scaled data agrees excellently with the $2 \mathrm{D}$ theoretical result, after estimating a very large Bohr radjus for the undoped single QW. ${ }^{16}$ Apparently there is contradiction for these two PL experiments. It is counterintuitive that $\mathrm{BGR}$ is scalable by $2 \mathrm{D}$ excitonic parameters and follows a universal behavior since $\mathrm{BGR}$ in quasi-2D is not only influenced by intrinsic material parameters. such as effective masses and dielectric constant, but aiso by the size of the structure because the screening of Coulomb potential is size-dependent, demonstrated by a form factor. ${ }^{17}$ In theory, many-body effects in semiconductor heterostructures and gain materials are quite successfully described in the framework of semiconductor Bloch equations (SBEs). ${ }^{18.19}$ Therefore. we will work with SBEs and take into account of the quasi-2D nature of the EHP in an $8 \mathrm{~nm} \mathrm{GaAs} / \mathrm{Al}_{0.3} \mathrm{Ga}_{0.7} . \mathrm{A}$ s single QW. And theoretical details are given in Section 2

Another physical quantity that is affected by carrier-carrier interaction is the effective mass. When the exchange interaction is screened efficiently. weak dependence of carrier self-energies on moment um leads to small mass reduction and increase in bandwidths. ${ }^{20}$ However. around transparency density. Coulomb enhancement is still important and

Further author information

Twas at Department of Electrical Engineering, Arizona State University.

J.Z.L.: E-mail: jianzhng@inas.nasa.gov

C.Z.N.: E-mail: cning@nas.nasa.gor 
change in the effective mass may not be negligible. As a matter of fact, recent time-resolved cyclotron resonance experiment $^{21}$ demonstrated a larger than ten percent change in the cyclotron mass of electrons in a photo-excited EHP in the bulk InSb, a narrow-bandgap semiconductor. The mass change is attributed to the enhanced $\vec{k} \cdot \vec{p}$ interaction as the bandgap is shrinked due to BGR. However, momentum-dependent exchange interaction contributes to the effective mass renormalization as well. Thus, it is worthy of looking into the problem to have a quantitative understanding. We adopt the same material system of an $8 \mathrm{~nm} \mathrm{GaAs} / \mathrm{Al}_{0.3} \mathrm{Ga}_{0.7} \mathrm{As}$ single $\mathrm{QW}^{-}$for this study.

As a consequence of EMR, the alpha factor, ${ }^{22,23}$ formally known as the linewidth enhancement factor, is expected to be modified accordingly. The factor is important for performance prediction of laser diodes since it affects the spectral linewidth, dynamical stability, pulse chirping, and beam quality. It is desirable to have as small an alpha factor as possible because it affects the maximum bit-rate in optical communication systems. ${ }^{24}$ Many-body theory is essential in the accurate characterization of spectral dependence of the alpha factor. ${ }^{25}$ We will show that mass renormalization does cause remarkable percentage change in the alpha factor at normal lasing carrier density; as compared to the conventional treatment of many-body effects without EMR. Section 2 outlines our two-band SBEs model and related formulation for calculation of EMR and the alpha factor. Numerical results and discussions are presented in Section 3, followed by the conclusions in Section 4.

\section{MODEL AND FORMULATION}

Semiconductor Bloch equations ${ }^{1,18,19}$ have been used to describe the coherent laser-semiconductor interaction with many-body effects. As the theory matures, various improvements are added to the pioneering work of Haug and Koch. ${ }^{1}$ Most notables are the inclusion of valence band structure and detailed dephasing calculation to get rid of the relaxation time approximation. ${ }^{2-28}$ As expected, semiconductor gain theory using SBEs becomes widely used in the pratice of modeling and simulation of optoelectronic devices. Normally an extra term, Coulomb hole correlation energy, is added to the carrier self-energy to account for the energy difference between a carrier in the presence of a bare Coulomb potential and a screened one within the Hatree-Fock approximation. Despite its non-self-consistent feature this mixed treatment allows accurate inclusion of many-body effects in the gain theory. ${ }^{19}$ Based upon the above consideration we choose this conventional treatment in the present work. The non-diagonal part of the semiconductor Bloch equations for a two-band electron-hole plasma is given as follows. in standard notations,

$$
\begin{aligned}
& {\left[\hbar \omega-\left(E_{g}+\Delta E_{g}+\epsilon_{\bar{k}}^{(e)}+\epsilon_{\vec{k}}^{(h)}-i \hbar \gamma_{p}\right)\right] P_{\vec{k}}=\left(f_{\vec{k}}^{(e)}+f_{\vec{k}}^{(h)}-1\right)\left[d_{\therefore: \vec{k}} E(\omega)-\sum_{\vec{k}^{\prime} \neq \vec{k}} r_{\vec{k}} \bar{k}_{\vec{k}} P_{\bar{k}^{\prime}}\right]} \\
& \Delta E_{g}=-\sum_{\overrightarrow{k^{\prime}} \neq \vec{k}} i_{\vec{k}^{\prime}-\vec{k}}^{s}\left[f_{\vec{k}^{\prime}}^{(e)}+f_{\vec{k}^{\prime}}^{(h)}\right]+\Delta E_{g}^{C H} \equiv \delta \epsilon^{(e)}-\dot{\alpha}^{(h)}+\Delta E_{g}^{C H} \text {. }
\end{aligned}
$$

In the above equations, other than the assumption of quasiequilibrium of the EHP. which means the distributions are solely determined by carrier density and temperature, as a consequence of the ultrafast scatterings among carriers, we need to model the screened Coulomb potential, $\gamma_{\vec{k}}^{s}$. In exact $2 \mathrm{D}$, an analvitical form, $T_{\vec{k}}^{-s p p}$. can be found for the potential under the so-called single plasmon pole approximation. ${ }^{1,3}$ In quasi-2D. a screening form factor. which measures the finite extension of the carrier wavefunctions along the confinement direction, has to be introduced in the screened potential. In the case where only the lowest subband is occupied. which is the case we are considering in this paper, the constant density of states leads to a weak dependence of the screeninc effect on carrier density. Near transparency density of a semiconductor QW: the relatively small screening length $\sim 25 \dot{A}$ allow's us to consider the long wavelength limit of the screening effect and a factorization of the screened porential into the screening form factor and 2D screened potential. The result is shown below.

$$
V_{\vec{k}}^{s}=F(\vec{k}) \Gamma_{\vec{k}}^{-s p p}, F(\vec{k})=\left(1+b_{0} k+b_{1} k^{2},^{-1},\right.
$$

where $b_{0}=2.67 \times 10^{-9}(\mathrm{~m})$ and $b_{1}=4.76 \times 10^{-19}\left(\mathrm{~m}^{2}\right)$. These two parameters are derive $\dot{c}$ as an extension to the single parameter fit to the form factor for an $8 \mathrm{~nm}$ GaAs QW.

Effective mass renormalization. as a manifestation of many-body effecis. is dut to both BGR-induced $\vec{k} \cdot \vec{p}$ interaction enhancement and momentum-dependence of the exchange contritution ir the carrier self-energy. In the present work, we focus on the momentum-dependent part (hereafter this part is referred to as exchange-induced 
EMR) and investigate the change in effective masses due to this part. The formulation is straight-forward as given below, by dropping carrier index for brevity,

$$
\frac{\delta m_{\mathrm{eff}}}{m_{\mathrm{eff}}}=-\frac{m_{\mathrm{eff}}}{m_{\delta}}, \frac{1}{m_{\delta}}=\frac{1}{\hbar^{2}} \cdot \frac{d^{2} \delta \varepsilon}{d k^{2}} .
$$

where $\delta$ denotes the corresponding exchange term in Eq. (2).

Finally, gain and carrier-induced phase shift are computed by matrix inversion of Eq. (1) and the alpha factor is defined as

$$
\alpha=-2 \frac{d^{2} \phi}{d N d z}\left(\frac{d G}{d N}\right)^{-1}
$$

following Chow et. al. ${ }^{25}$ Comparison is made between cases with and without effective mass renormalization. For the latter case, rigid shift of the carrier self-energies by BGR is considered. Fig.(1) show's our numerical results of gain and carrier-induced phase shift with and without EMR.

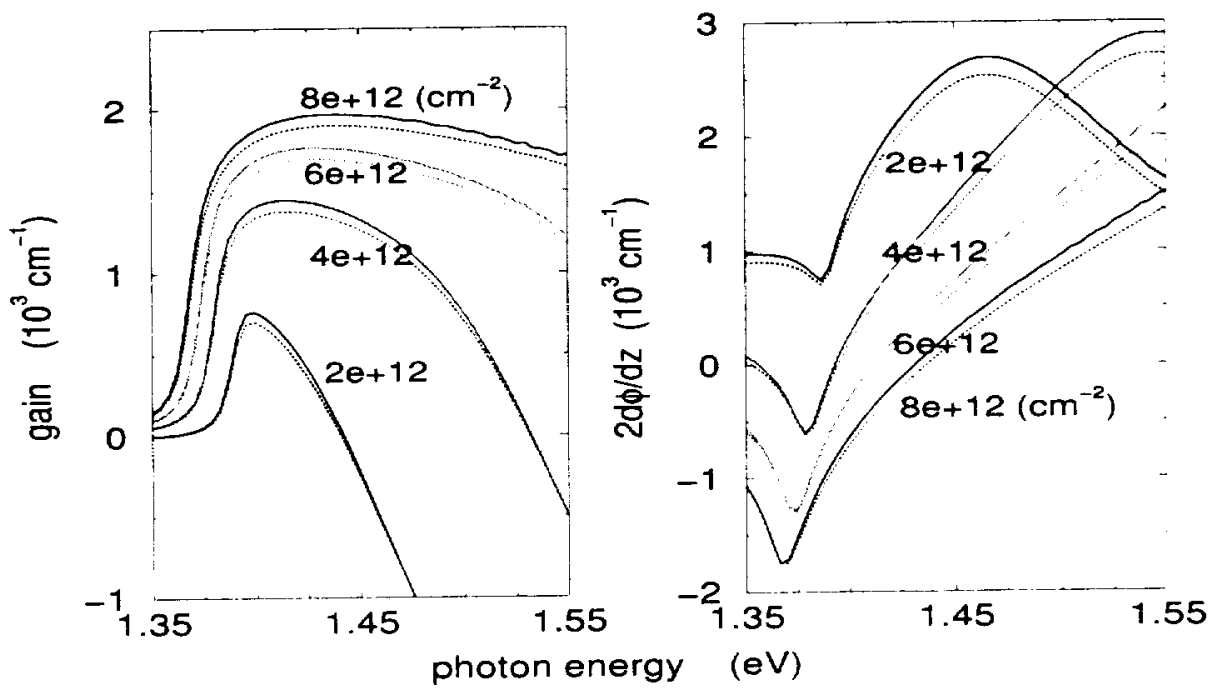

Figure 1. Gain and carrier-induced phase shift results as obtained by matrix inversion of the SBE for an $8 \mathrm{~nm}$ GaAs QW. Bandgap shrinkage is clearly seen as curves red-shift when carrier density increases. Solid curves take into account of effective mass renormalization and the dotted curves do not.

\section{RESULTS AND DISCUSSIONS}

We restrict our studied system solely to $8 \mathrm{~nm} \mathrm{GaAs} / \mathrm{Al}_{0.3} \mathrm{Ga}_{0.7} \mathrm{As}$ single $\mathrm{QWs}$ at $300 \mathrm{~K}$ and with areal plasma densities in the range of $1 \times 10^{12} \mathrm{~cm}^{-2}$ to $8 \times 10^{12} \mathrm{~cm}^{-2}$. The dephasing rat $\epsilon$. $\hbar \gamma_{p}$, is taken to be 5 meV. Material parameters can be easily found in literature and will not be given here.

\subsection{Bandgap shrinkage}

Bandgap renormalization in electron-hole plasma in both $2 \mathrm{D}^{5}$ and $3 \mathrm{D}^{29}$ has been known to follow scaling law's, though there are disagreements in quasi-2D. ${ }^{10}$ Photoluminescent spectroscopy has been utilized in determination of BGR in photoexcited EHP systems, and experimental data vary from group to group i.9.11.13.15 Theoretically. Jalabert and Das Sarma ${ }^{10}$ have compared their BGR result. calculated with dynamical :indom-phase-approximation and scaled with $2 D$ excitonic parameters. with the experimental data by. Tränkle et. al.. ${ }^{-8}$ which suggested a universal scaling law for BGR in quasi-2D semiconductor QW structures when scaled with the same $2 \mathrm{D}$ excitonic parameters. and found no agreement. 
In Fig.2, our numerical results of BGR in an $8 \mathrm{~nm} \mathrm{GaAs} / \mathrm{Al}_{0.3} \mathrm{Ga}_{0.7} \mathrm{As}$ single $\mathrm{QW}$ is compared with scaling behaviors in both 2D and 3D. We have adopted an excitonic Rydberg $(R y)$ of 9 mel for our quasi-2D system ${ }^{30}$ and the Bohr radius $\left(a_{0}\right)$ has been computed to be $84.76 \AA$ by assuming $a_{0}^{2} \times R y=$ const for a specific material system. A fitting expression for BGR has been found to be

$$
\Delta E_{g} / R y=-2.31382 \times r_{s}^{-0.7137}, r_{s}=\left(\pi n a_{0}^{2}\right)^{-\frac{1}{2}},
$$

Also plotted in the figure are experimental data by Jones et. al. ${ }^{15}$ scaled with the same parameters. Apparently there is no agreement among any of these curves. It is worth noting that our data move up above the $2 \mathrm{D}$ curve if scaled by 2D parameters, so do the experimental data by Jones et. al., but the data by Tränkle et. al., if scaled by quasi-2D parameters, lie below the 3D curve. A consistent understanding of our data, in comparison with scaling laws in 2D and 3D, lies in the screening capability of the EHP. As the system undergoes quantum confinement in a certain direction its efficiency of screening is reduced accordingly. ${ }^{8}$ So, even though Coulomb interaction is enhanced at reduced dimensionality, as indicated by larger excitonic Rydberg energy: the scaled BGR curves lie above 3D's. It is important to point out that this reduction in screening efficiency depends upon the size of the QW structure and a smooth behavior is expected since no abrupt change in any parameter has been found. This would lead us to conclude that there is no universal scaling behavior for BGR in semiconductor QW structures. Furthermore, it is interesting to note that a fully quantum mechanical interpretation of PL spectroscopy with many-body effects has been discussed by Koch et. al. ${ }^{31}$ in this symposium, which may shed light upon resolving the high divergence of the PL data from different groups.

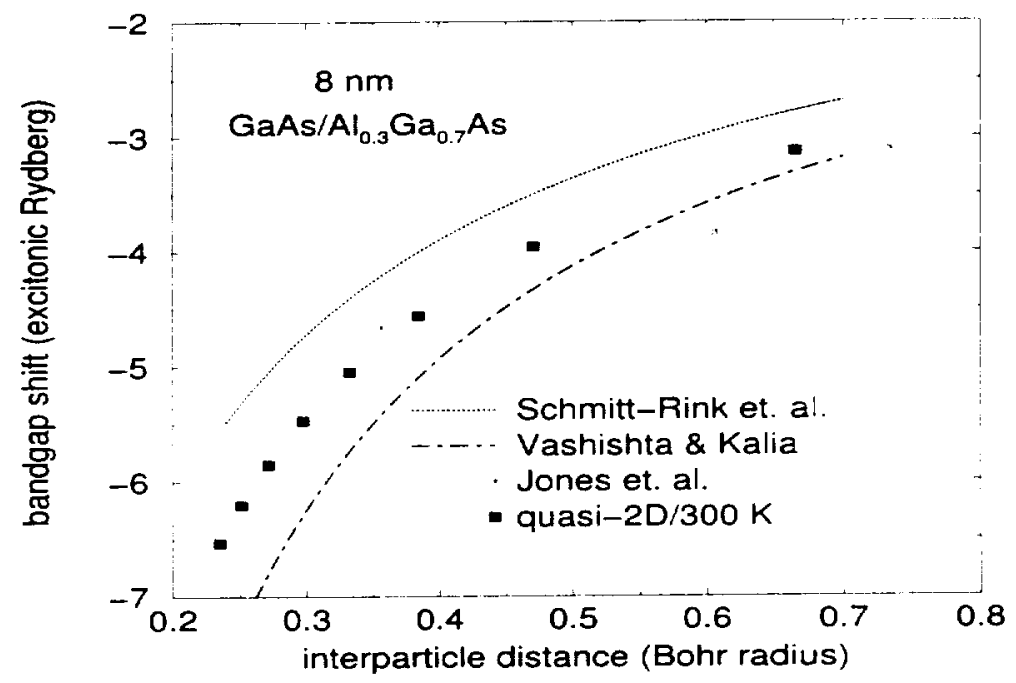

Figure 2. Numerical results of bandgap shrinkage for an $8 n m \mathrm{GaAs} Q W$, as compared with 2D analy tical expression.

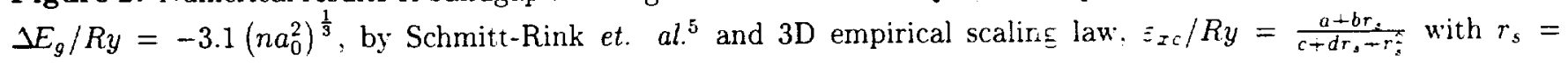
$\left(\pi n a_{0}^{2}\right)^{-\frac{1}{2}}$, by Vashishta and Kalia. ${ }^{29}$ Also shown are experimental data by Jones et. al., ${ }^{15}$ scaled by the quasi-2D excitonic parameters. No universal scaling behavior in quasi-2D is vindicatec.

\subsection{Effective mass renormalization}

Effective mass renormalization due to momentum-dependent exchange interaction is negative at low energy and relatively small, as shown in Fig.3. The percentage change in the effective mass can reach over $8 \%$ for holes near $\Gamma$ point at a density of $6 \times 10^{12} \mathrm{~cm}^{-2}$. while electrons are only about $3 \%$ lighter a- maximum at $1 \times 10^{12} \mathrm{~cm}^{-2}$. Exchangeinduced EMR is reduced as carriers move away from $\Gamma$ point. And at high energy; exchange-induced EMR is reversed and carriers become heavier, though the percentile is smaller. As carrier density increases, exchange-induced EMR reduces for electrons. The cause is a mixture of increasing screening and inefficient occupation of the added electrons, 
as explained below. Coulomb potential is weaker as screening becomes stronger, and added electrons fill high energy states near the chemical potential, due to electrons' statistical degeneracr. The exchange interaction still increases, but its momentum dependence is effectively weaker because the added electrons interact weaker at high energy (i.e., large momentum), according to Eq. (2), as both the potential and distribution function are smaller in magnitude at large momentum. Therefore, exchange-induced EMR decreases with the carrier density. Holes basically follow a Maxwell-Boltzmann distribution so the added holes spread over the energ. spectrum. As a result, the exchange interaction steadily increases with carrier density. Therefore, its exchange-induced EMR monotonically grows with carrier density.

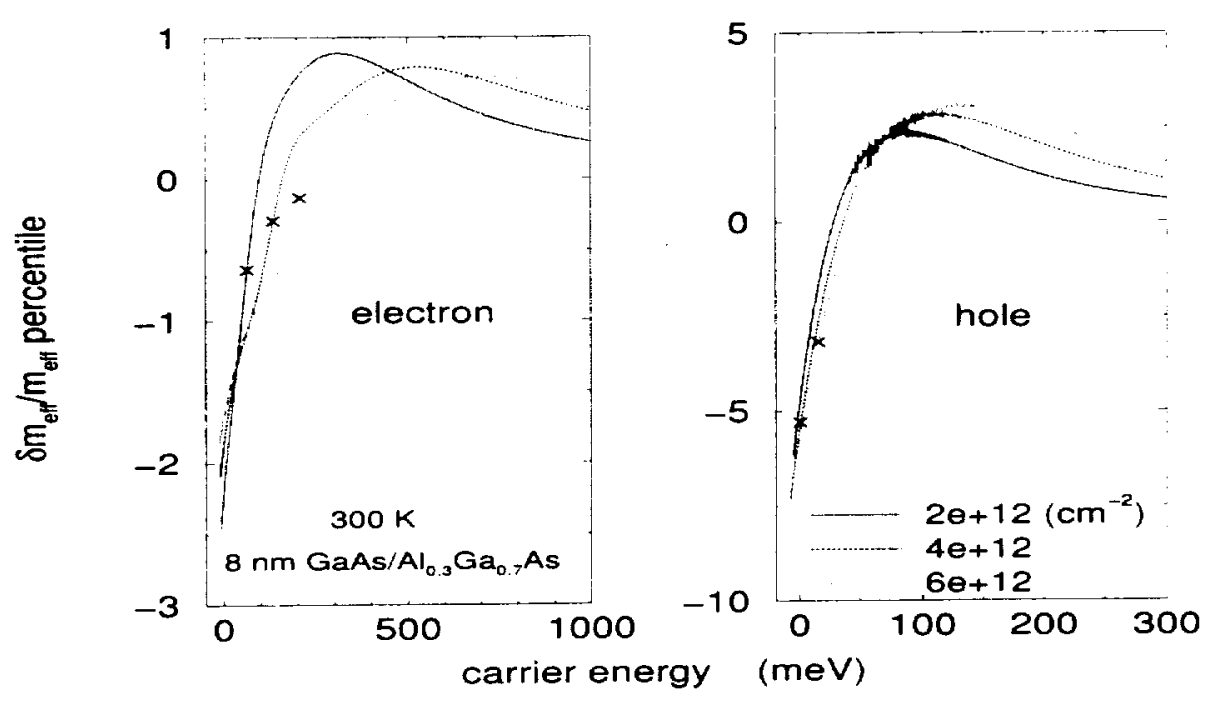

Figure 3. Energy dependence of percentage change in the effective mass due to momentum-dependent exchange interaction for electrons and holes. Positions of the chemical potential are marked with crosses. The change is maximum in magnitude at $\Gamma$ point for both electrons and holes, and approaches zero at large energy: Carriers can be heavier in certain energy range bevond the chemical potential.

Depicted in Fig. 4 are the dependence of percentage change in the effective mass upon carrier density for electrons at both $\Gamma$ point and the chemical potential and for holes at $\Gamma$ point. Because of their smaller mass. electrons experience a markedly different change in exchange-induced EMR in contrast to what holes go through. The momentum-dependent exchange interaction makes electrons' mass lighter as density drops. However. EMR due to BGR-induced enhanced $\vec{k} \cdot \vec{p}$ interaction could overhelm it from distinction, especially in narrow-bandgap semiconductors. Subsequent work will clarify this point. The change at the chemical potential is smaller because of weaker exchange interaction at larger energy: It is worth mentioning that Jalabert and Das Sarma ${ }^{10}$ found similar results for the effective mass renormalization in quasi-2D electron gases by using dynamical random-phast-approximation, as shown in their Fig.3.

Now let's discuss the time-dependent behavior of the electrons' cyclotron mass in narrow-bandgap InSb studied by Kono et. al. ${ }^{21}$ in an interband-pump-and-intraband-probe experiment, withis our two-band model. They found that the electron effective mass "increases as a function of time." In a typical time-resorled photoexcitation experiment, ultrafast thermalization is followed by cooling down of carriers through interaction with LO phonons. Quasiequilibrium can be assumed after ultrafast thermalization. Then spontaneous emissions at a nanosecond timescale start to take place, which is a slow channel to consume carriers. The time delay in the experiment extends to $1.2 \mathrm{~ns}$. The fast change in the cyclotron mass. in the order of $100 \mathrm{ps}$, if to be related to density decline. is most probably through Auger processes, which have been known to dominate carrier consumption in long wavelength interband lasers. Further study is needed in order to fully understand the EMR behicior in the experiment. The doninant EMR contribution in InSb is due to BGR-induced enhanced $\vec{k} \cdot \vec{p}$ interaction. which has been estimated to be about $17 \%$ and exchange-induced EMR is a minor correction to the $\vec{k} \cdot \vec{p}$ contribution. 


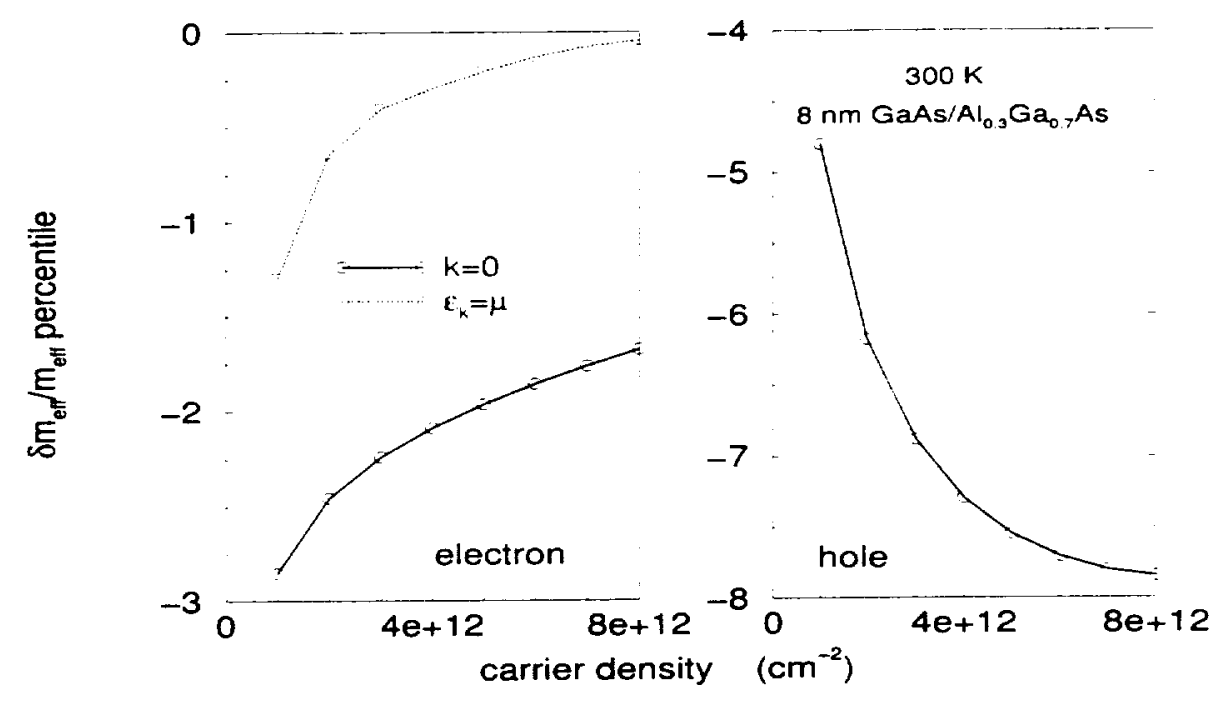

Figure 4. Carrier density dependence of percentage change in the effective mass due to momentum-dependent exchange interaction at $\Gamma$ point (solid line), and at chemical potential (dotted line) for electrons and at $\Gamma$ point for holes. Electrons' change due to exchange interaction increases as density drops in the studied range because of their statistical degeneracy, in contrast to holes' change.

\subsection{The alpha factor}

As important a parameter as the alpha factor, the suppression of it is what technologists dream of. The factor has been reduced considerably by using quantum well structure.$^{32}$ Reduced density of states at reduced dimensionality allows easier population inversion (i.e., lower threshold) and larger differential gain. As EMR occurs, the lighter mass means further decrease in the density of states. Therefore we expect an additional reduction in the alpha factor. In Fig.5, percentage change in the alpha factor due to exchange-induced EMR is shown. The change is nonnegligible and increases with carrier density. At certain photon energy it can reach a 20 percent suppression. However. the change at the peak gain frequency is uncertain and relatively small.

\section{CONCLUSIONS}

Within the framework of semiconductor Bloch equations with many-body effects under the Hatree-Fock approximation and the single plasmon pole approximation for static screening, we have demonstrated that there is no universal scaling law for the bandgap renormalization in semiconductor quantum well structures. The phrsical reason is simply because the screening effect is dependent upon QW size, which promises a smooth transition from 3D to $2 \mathrm{D}$. The effective mass renormalization due to momentum-dependent exchange interaction can be as large as 8 percent for holes and smaller for electrons, while its dependence on carrier density relies on the degree of statistical degeneracy of carriers. In the range of interest, $1 \times 10^{12}$ to $8 \times 10^{12} \mathrm{~cm}^{-2}$, exchange-induced EMR drops for electrons as carrier density increases. in marked contrast with holes. As for the alpha factor, it $\leqslant$ relative change increases with carrier density and can be as large as 20 percent near its minimum at the highest density of $8 \times 10^{12} \mathrm{~cm}^{-2}$ in our study: However, its change at the peak gain frequency is not as remarkable.

\section{ACKNOWLEDGMENTS}

This work is supported by the Director's Discretionary Fund of the NASA Ames Research Center under NASA contract NAS2-14303. 


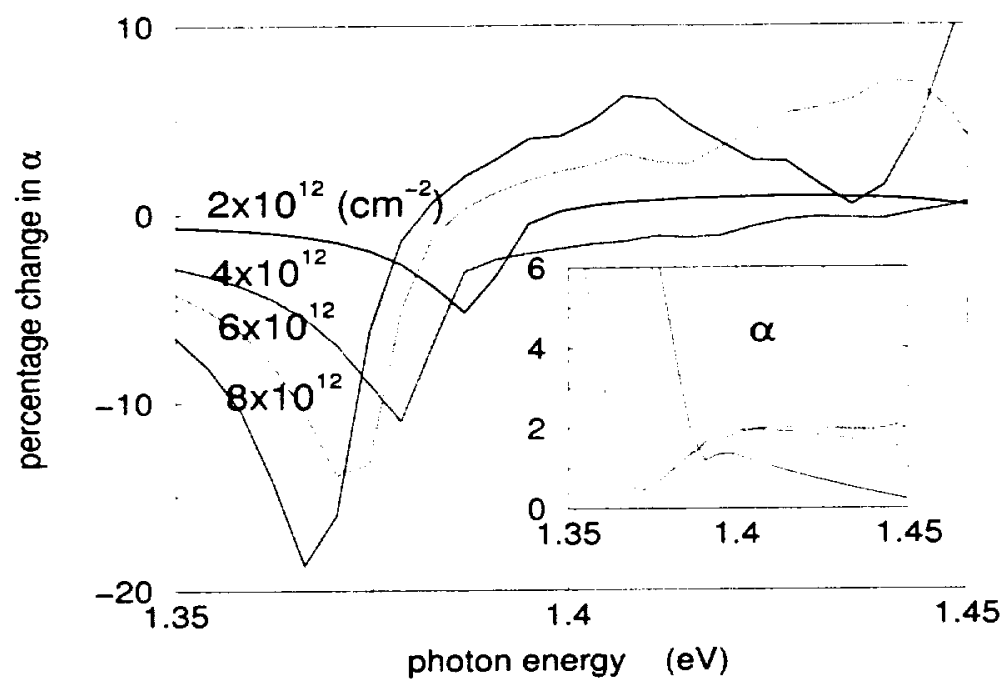

Figure 5. Percentage correction to the linewidth enhancement factor due to effective mass renormalization. The correction increases with carrier density. Inset shows the calculated alpha factor. Solid lines are with EMR and dotted lines are without.

\section{REFERENCES}

1. H. Haug and S. W. Koch, "Semiconductor laser theory with many-body effects," Phys. Rer. A39, pp. 188i-1898, 1989.

2. H. Haug and S. Schmitt-Rink, "Electron theory of the optical properties of laser-excited semiconductors," Prog. Quantum Electron. 9. pp. 3-100, 1984.

3. S. Schmitt-Rink, C. Ell, and H. Haug, "Many-body effects in the absorption, gain. and luminescence spectra of semiconductor quantum-well structures," Phys. Rev. B33, pp. 1183-18\$9, 1986.

4. S. Schmitt-Rink and C. Ell, "Excitons and electron-hole plasma in quasi-two-dimensional system." J. Lumin. 30, pp. 585-596, 1985.

5. S. Schmitt-Rink, D. S. Chemla, and D. A. B. Miller, "Linear and nonlinear optical properties of semiconductor quantum wells," Adv. Phys. 38. pp. 89-188, 1989.

6. D. A. Kleinman and R. C. Miller, "Band-gap renormalization in semiconductor quantum wells containing carriers," Phys. Rev. B32, pp. 2266-22ґ2, 1985.

7. G. Tränkle, E. Lach, A. Forchel, F. Scholz, C. Ell, H. Haug, G. Weimann. G. Griffiths, H. Kroemer. and S. Subbanna. "General relation between band-gap renormalization and carrier density in two-dimensional electron-hole plasmas," Phys. Rev. B36, pp. 6712-6714, 1987.

8. G. Tränkle, H. Leier, A. Forchel, H. Haug, C. Ell, and G. Weimann, "Dimensionality dependence of the band-gap renormalization in two- and three-dimensional electron-hole plasmas in GaAs," Phys. Rev. Lett. 58. pp. 419-422. 1987.

9. C. Delalande. G. Bastard, J. Orgonasi. J. A. Brum, H. W. Lin, M. Yoos, G. Weimann, and W. Schlapp. "Many-body effects in a modulation-doped semiconductor quantum well." Phys. Rev. Lett. 59. pp. 2690-2692. $198 \pi$.

10. R. Jalabert and S. D. Sarma, "Mnay-body effects in GaAs-based two-dimensional electron systems." Surf. Sci. 229. pp. 405-409, 1990.

11. M. Potemski. J. C. Mann. K. Ploog. and G. Weimann, "High intensity excitation luminescence of quant um wells in high magnetic fields." Surf. Sci, 229. pp. 380-383, 1990.

12. M. Potemski. J. C. Mann, K. Ploog, and G. Weimann, "Properties of a dense quasi-two-dimensional electron-hole gas at high magnetic fields," Solid State Comm. 75, pp. 185-188, 1990. 
13. T. L. Reinecki, D. A. Broido, E. Lach, V. Kulakovskii, A. Forchel, and D. Gruetzmacher, "Bandgap renormalization at finite carrier densities in semiconductor quantum wells and mesa structures," Superlattices and Microstructures 7, pp. 437-440, 1990.

14. R. Congolani, G. C. L. Rocha, H. Kalt, K. Ploog, M. Potemski, and J. C. Mann. "Magnetoluminescence of the two-dimensional electron-hole fluid," Phys. Rev. B43, pp. 9662-9671. 1991.

15. E. D. Jones, M. Blount, W. Chow, H. Hou, J. A. Simmons, Y. Kim, and T. Schmiedel, "Bandgap renormalization: GaAs/AlGaAs quantum wells," in Physics and Simulation of Optoelectronic Devices VI. M. Osiński. P. Blood. and A. Ishibashi, eds., Proc. SPIE 3283, pp. 350-356, 1998.

16. Jones et. al. estimated an $a_{0} \sim 12.5 \mathrm{~nm}$ for the $8 \mathrm{~nm}$ undoped GaAs/AlGaAs single quantum wells, which is larger than the 3D Bohr radius $(\sim 12 \mathrm{~nm})$.

17. G. Bastard, Wave mechanics applied to semiconductor heterostructures. Halsted. New York, 1988.

18. H. Haug and S. W. Koch, Quantum Theory of the Optical and Electronic Properties of Semiconductors, World Scientific, Singapore, 1994.

19. W. W. Chow and S. W. Koch, Semiconductor-Laser Fundamentals: Physics of the Gain Materials, Springer, Berlin, 1999.

20. To the contrary, Schmitt-Rink et. al. ${ }^{3}$ indicated mass enhancement and decrease in bandwidths.

21. J. Kono, A. H. Chin, A. P. Mitchell, T. Takahashi, and H. Akiyama, "Picosecond time-resolved cyclotron resonance in semiconductors," Appl. Phys. Lett. 75, pp. 1119-1121, 1999.

22. H. Haug and H. Haken, "Theory of noise in semiconductor laser emission," Zeitschrift für Physik 204, pp. 262$275,1967$.

23. C. H. Henry, "Theory of the linewidth of semiconductor lasers," IEEE J. Quantum Electron. QE-18, pp. 259$264,1982$.

24. G. P. Agrawal and N. K. Dutta, Long-Wavelength Semiconductor Lasers, Van Nostrand. New York, 1986.

25. W. W. Chow, S. W. Koch, M. S. III, and C. Ell, "Many-body effects on the linewidth enhancement factor in quantum well lasers," Appl. Phys. Lett. 58, pp. 328-330, 1991.

26. S. Hughes, A. Knorr, S. W. Koch, R. Binder, R. Indik, and J. V. Moloney, "The influence of electron-hole scattering on the gain spectra of highly excited semiconductors," Solid State Comm. 100. pp. 555-559, 1996.

27. C. Z. Ning, R. A. Indik, J. V. Moloney, W. W. Chow, A. Girndt. S. W. Koch. and R. Binder, "Incorporating many-body effects into modeling of semiconductor lasers and amplifiers," in Physics and Simulation of Optoelectronic Devices V, M. Osiński and W. W. Chow, eds., Proc. SPIE 2994. pp. 666-676, 1997.

28. A. Girndt, S. W. Koch, and W. W. Chow, "Microscopic theory of laser gain in semiconductor quantum wells," Appl. Phys. A66, pp. 1-12, 1998.

29. P. Vashishta and R. K. Kalia, "Lniversal behavior of exchange-correlation energy" in electron-hole liquid," Phys. Rev. B25, pp. 6492-6495, 1982.

30. R. L. Green and K. K. Bajaj. "Binding energies of wannier excitons in GaAs-Ga $a_{(1-x}, A l_{(x)}$ As quantum-well structures in a magnetic field," Phys. Rev. B31, pp. 6498-6502, 1985.

31. S. W. Koch, M. Kira, and F. Jahnke, "Microscopic theory of semiconductor light emission," in Physics and Simulation of Optoelectronic Devices VIII, R. Binder, P. Blood, and M. Osiński, eds., Proc. SPIE 3944. pp. --.--..., 2000.

32. Y. Arakawa and A. Yariv, "Theory of gain, modulation response, and spectral linewidth in AlGaAs quantum well lasers," IEEE J. Quantum Electron. 21, pp. 1666-1674, 1985. 